\author{
R. P. Stolk · P. Suriyawongpaisal $\cdot$ W. Aekplakorn • \\ M. Woodward $\cdot$ B. Neal $\cdot$ for the InterASIA \\ Collaborative Group*
}

\title{
Fat distribution is strongly associated with plasma glucose levels and diabetes in Thai adults-the InterASIA study
}

Received: 2 July 2004 / Accepted: 7 November 2004 / Published online: 3 March 2005

C) Springer-Verlag 2005

\begin{abstract}
Aims/hypothesis: Asian populations have high risks of disease at low levels of BMI and weight, possibly because of high rates of abdominal obesity. In such populations, waist circumference and WHR (measures of fat distribution) may better capture the effects of adiposity. Methods: The strengths of the associations between different measures of adiposity and glucose levels and diabetes were investigated in the Thai component of the International Collaborative Study of Cardiovascular Disease in Asia (InterASIA), a multi-stage cross-sectional survey of risk factors in Thai adults aged 35 years or over. The analyses included 5,302 men and women. Results: All four measures of adiposity were positively associated with plasma glucose and the odds of having diabetes (all $p<0.001$ ), but the associations were stronger for measures of fat distribution. The age- and sex-adjusted fasting plasma glucose level increased linearly across each fifth of weight, BMI, waist and WHR by $0.12 \mathrm{mmol} / 1$ (SE 0.02 ), 0.12 (0.02), $0.17(0.02)$ and $0.16(0.02)$, respectively. The corresponding odds ratios for diabetes were 1.41 (95\% CI $1.27-1.56), 1.43$ (1.28-1.59), $1.64(1.47-1.83)$ and 1.70 (1.52-1.90), respectively. Multivariate analyses incorporating different combinations of adiposity measures, as well
\end{abstract}

*Members listed in Acknowledgements

R. P. Stolk $(\bowtie)$

Julius Center for Health Sciences and Primary Care,

University Medical Center Utrecht,

Str 6.131,

PO Box 85500, 3508 GA Utrecht, The Netherlands

e-mail: r.p.stolk@jc.azu.nl

Tel.: +31-30-2509305

Fax: +31-30-2505485

R. P. Stolk · M. Woodward - B. Neal

The George Institute for International Health,

University of Sydney,

Australia

P. Suriyawongpaisal · W. Aekplakorn

Community Medicine Center, Faculty of Medicine,

Ramathibodi Hospital, Mahidol University,

Bangkok, Thailand as analyses of receiver operating characteristics, confirmed the greater predictive value of measures of fat distribution. Conclusions/interpretation: Waist circumference and WHR were more strongly associated with fasting plasma glucose and diabetes than were weight and BMI. These measures of abdominal adiposity are likely to be more useful for assessing the obesity-related risk of cardiovascular diseases in Asian populations.

Keywords Blood glucose $\cdot$ Body fat distribution Obesity · Thailand - Waist circumference

Abbreviations InterASIA: International Collaborative Study of Cardiovascular Disease in Asia - ROC: Receiveroperator curve $\cdot$ WHO: World Health Organization

\section{Introduction}

Obesity, and in particular abdominal obesity, is a growing public health problem in Asia, where it is associated with an escalating prevalence of type 2 diabetes, hypertension and cardiovascular disease [1]. Furthermore, the burden of obesity-related disease in Asia appears disproportionate to the relatively low prevalence of obesity as defined by World Health Organization (WHO) criteria [2]. For the same BMI, Asian populations appear to show higher levels of risk factors and disease than their European counterparts [3]. This may be because current WHO cut-off points for obesity are set too high for Asian populations [4]. Alternatively, it is possible that the associations of obesity with risk factors and disease may be different in Asian populations, perhaps as a consequence of the varied effects of different patterns of fat distribution.

In 2000, the International Collaborative Study of Cardiovascular Disease in Asia (InterASIA) was conducted in China and Thailand. Its goals were to estimate the prevalence and distribution of cardiovascular disease risk factors in a representative sample of the adult population [5]. This is one of the few population-based studies in Asia that included measurements of weight, height, waist circum- 
ference and hip circumference. We used data from the Thai participants to investigate which measure of adiposity best predicted high glucose levels and the presence of diabetes.

\section{Subjects, materials and methods}

InterASIA was conducted by academic institutions in Australia, China, Thailand and the United States of America (see Acknowledgements). The Thai component of the study was approved by the Institutional Review Board at Mahidol University, Bangkok and by the local ethics committee at each of the collaborating centres. All participants provided written, informed, consent and the study was conducted in line with the Declaration of Helsinki and subsequent amendments.

The sample design The sample design has been described in detail previously [5]. In brief, one representative province was selected from each of the four main geographic regions of Thailand. From these provinces and Bangkok, a total of five representative urban districts (one from Bangkok and one from each chosen province) and four representative rural districts (one from each chosen province) were selected. Up to six representative enumeration districts were selected from each district. Within the selected enumeration districts, the population aged 35 years and over was grouped by age (35-44, 45-54, 55-64 and $\geq 65$ years) and sex. Individuals were then sampled at random from each age and sex group with the goal of recruiting a similar number of people from each age group and sex group, with the proviso that only one individual be recruited per household.

Data collection and measurements For each individual who agreed to participate, trained study staff went through a structured questionnaire, performed a brief physical examination and collected a fasting venous blood sample. Anthropometric measures were performed while the par- ticipant was standing, wearing indoor clothes without shoes. Waist circumference was measured in the horizontal plane $1 \mathrm{~cm}$ above the navel, whereas hip circumference was measured at the level of the maximal protrusion of the gluteal muscles. Diabetes was defined as a fasting plasma glucose value of $7.0 \mathrm{mmol} / 1$ or more, or a previous diagnosis of diabetes (but not a previous diagnosis of gestational diabetes).

Statistical methods Associations between both glucose and diabetes and the four measures of adiposity were assessed as age- and sex-adjusted linear effects across the sex-specific fifths of the adiposity variables. For glucose this involved use of general linear models, whilst for diabetes logistic regression models were fitted. Subjects with known diabetes mellitus were excluded from all analyses based on plasma glucose values. Receiver-operator curve (ROC) analyses were performed to assess how well each measure of adiposity predicted the presence of diabetes. All analyses allowed for the complex survey design, using STATA 7.0 statistical software (StataCorp, College Station, TX, USA). Enumeration districts were taken as primary sampling units, age and sex as strata and weights were the population : sample size ratios for each combination of age, sex and type of enumeration district. We considered $p$ values smaller than 0.05 to be statistically significant.

\section{Results}

The characteristics of the study population are summarised for men and women in Table 1. Using the recently proposed definitions for Asian populations [4], the prevalence of all adiposity measures was higher in women than in men.

Associations of obesity with fasting plasma glucose All measurements of obesity were positively associated with fasting plasma glucose levels, independent of age and sex

Table 1 Clinical characteristics of the study population

\begin{tabular}{|c|c|c|c|c|c|c|c|}
\hline & \multicolumn{3}{|c|}{ Men $(n=2093)$} & \multicolumn{3}{|c|}{ Women $(n=3209)$} & \multirow{2}{*}{$\begin{array}{l}\text { Difference } \\
\text { (men-women) }\end{array}$} \\
\hline & Mean & $(\mathrm{SE})$ & Inter-quintile range & Mean & $(\mathrm{SE})$ & Inter-quintile range & \\
\hline Weight (kg) & 61.4 & $(0.8)$ & $51.6-70.6$ & 57.6 & $(0.5)$ & $48.7-65.5$ & $p<0.001$ \\
\hline BMI $\left(\mathrm{kg} / \mathrm{m}^{2}\right)$ & 23.1 & $(0.2)$ & $19.8-25.9$ & 24.8 & $(0.2)$ & $21.3-28.1$ & $p<0.001$ \\
\hline Waist circumference $(\mathrm{cm})$ & 80.8 & $(0.9)$ & $70.2-90.7$ & 80.7 & $(0.7)$ & $70.5-90.1$ & $p=0.9$ \\
\hline WHR & 0.89 & $(0.01)$ & $0.83-0.95$ & 0.86 & $(0.01)$ & $0.79-0.93$ & $p=0.9$ \\
\hline Age (years) & 50.3 & \multicolumn{2}{|l|}{$(1.2)$} & 50.9 & $(1.2)$ & & $p=0.7$ \\
\hline Fasting plasma glucose (mmol/l) & 5.60 & \multicolumn{2}{|l|}{$(0.08)$} & 5.50 & (95\% Confidence interval) & onfidence interval) & $p=0.3$ \\
\hline Overweight (BMI $\geq 23 \mathrm{~kg} / \mathrm{m}^{2}$ ) & $46.0 \%$ & \multicolumn{2}{|c|}{$(41.2-51.0)$} & $63.7 \%$ & \multicolumn{2}{|c|}{$(59.8-67.5)$} & $p<0.001$ \\
\hline Obesity $\left(\mathrm{BMI} \geq 27.5 \mathrm{~kg} / \mathrm{m}^{2}\right)$ & $11.2 \%$ & \multicolumn{2}{|c|}{$(8.9-14.2)$} & $22.7 \%$ & \multicolumn{2}{|c|}{$(19.9-25.9)$} & $p<0.001$ \\
\hline $\begin{array}{l}\text { Abdominal obesity (waist } \\
\text { circumference }>85 \mathrm{~cm} \text { in men, } \\
>80 \mathrm{~cm} \text { in women) }\end{array}$ & $34.9 \%$ & \multicolumn{2}{|c|}{$(28.2-42.2)$} & $50.7 \%$ & \multicolumn{2}{|c|}{$(45.5-56.0)$} & $p<0.001$ \\
\hline Diabetes mellitus & $9.1 \%$ & \multicolumn{2}{|c|}{$(7.4-11.2)$} & $10.0 \%$ & \multicolumn{2}{|c|}{$(8.4-11.9)$} & $p=0.5$ \\
\hline
\end{tabular}


Fig. 1 Proportionate mean glucose level $(95 \%$ confidence bars) by fifths, for each measure of adiposity. For each measure, proportions and confidence limits were calculated by dividing the mean plasma glucose level (adjusted for age and sex) and confidence limit in each fifth by the adjusted mean in the first fifth (taken as the reference level)

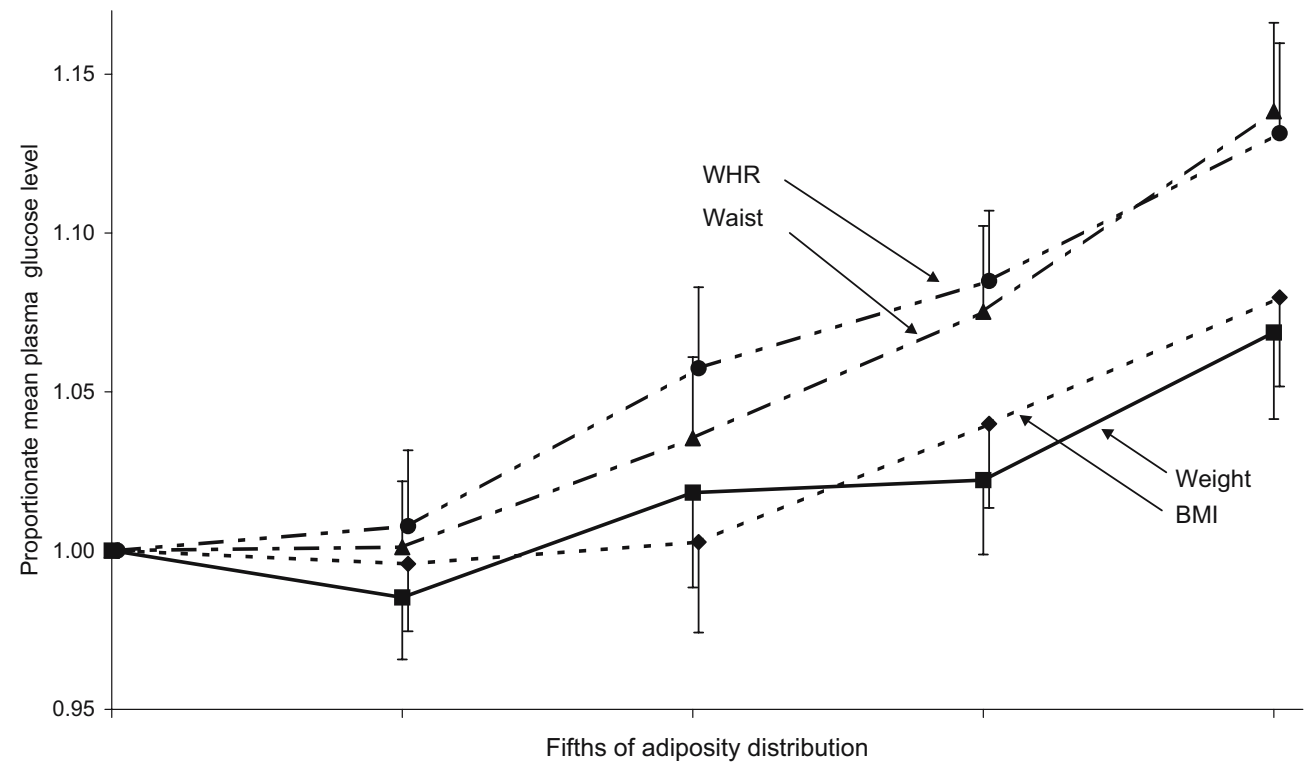

all $p<0.001$, Fig. 1). The associations were stronger for measures of fat distribution (weight, WHR) than for weight or BMI. The age- and sex-adjusted plasma glucose level increased linearly across each fifth of weight, BMI, waist circumference and WHR by $0.12 \mathrm{mmol} / \mathrm{l}$ (SE 0.02), 0.12 (0.02), $0.17(0.02)$ and $0.16(0.02)$, respectively (all $p<$ $0.01)$. If both BMI and waist circumference were included in the same model, the association between BMI and glucose level was no longer statistically significant and the association between waist circumference and glucose level was strengthened. The same pattern was observed in models including BMI and WHR. Likewise, in models in which

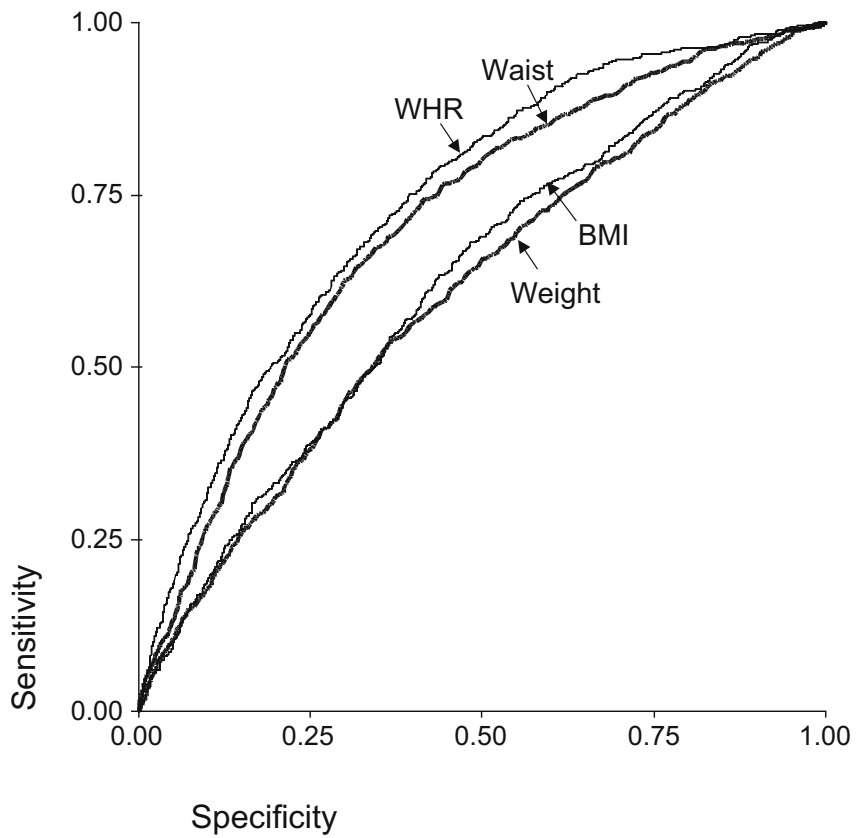

Fig. 2 Receiver operator curves for diabetes and each measure of adiposity. Test for differences in AUC: WHR or waist circumference versus BMI or weight. $p<0.001$ for all values waist circumference or WHR were included with weight the association with the measure of fat distribution was strengthened and the association with weight weakened.

Diabetes mellitus All measures of adiposity were greater in participants with diabetes mellitus than in those without diabetes: adjusted for age and sex, the differences were 2.17 (SE 0.8) $\mathrm{kg}$ for weight, $0.9(0.3) \mathrm{kg} / \mathrm{m}^{2}$ for BMI, 3.1 $(0.8) \mathrm{cm}$ for waist circumference and $0.03(0.005)$ units for WHR (all comparisons $p<0.001$ ). Similarly to plasma glucose, the associations of diabetes with adiposity were stronger for waist circumference and WHR than for weight and BMI. The age- and sex-adjusted odds ratios for diabetes per fifth of the distribution were 1.41 for weight (95\% CI 1.27-1.56), 1.43 for BMI (1.28-1.59), 1.64 for waist circumference (1.47-1.83) and 1.70 for WHR (1.521.90). Analogous to the findings for plasma glucose, the addition of a measure of fat distribution (waist circumference or WHR) to a model of the association of either BMI (or weight) with diabetes resulted in a diminished association of BMI (or weight) with diabetes and strengthened associations for the measures of fat distribution. The areas under the ROC for BMI and weight were 0.69 , compared to 0.75 for WHR and waist (Fig. 2). The differences in area between BMI (or weight) and each measure of fat distribution were all highly statistically significant (all $p<$ $0.001)$. Repeating the ROC analyses in men and women separately produced similar results.

\section{Discussion}

These analyses clearly demonstrate that among Thai adults, waist circumference and WHR were more strongly associated with fasting plasma glucose and diabetes than either weight or BMI. Given the established strong associations of diabetes and impaired fasting glucose with the risk of cardiovascular disease, these findings suggest that in Thai 
populations a simple measure of fat distribution is likely to be a better indicator of risk than the measures of obesity more typically employed. This finding is supported by studies in Caucasian populations that have shown that intraabdominal fat is a more important determinant of cardiovascular risk than overall obesity [6]. The finding is also consistent with studies that have highlighted the importance of central adiposity as a risk factor in Asian populations $[2,3,7,8]$. However, these analyses are not based on a gold standard assay of intra-abdominal fat such as magnetic resonance imaging, and measuring waist circumference is often less practical than measuring weight.

A particularly important feature of the current findings is that the associations of each of the measures of adiposity with glucose levels and the risk of diabetes were continuous across a broad range of adiposity measures extending well below levels currently used by the WHO to define overweight and abdominal obesity. As such, these data suggest that current cut-off points for overweight and obesity may be poor indicators of obesity-related risk in Asian populations. Alternative means of evaluating obesity-related risk based on continuous measures of central adiposity would provide much better information. Alternatively, redefined lower cut-off points to define overweight and obesity using measures of central adiposity (waist circumference or WHR rather than BMI or weight) might be a less good but more practical compromise $[2,4]$.

Obesity and overweight have now replaced under-nutrition and micronutrient deficiencies as the leading causes of disease attributable to diet in most Asian countries [9]. In dealing with these new epidemics of non-communicable disease, public health services require quantitatively reliable information about the magnitude of the risks caused by obesity. Our data suggest that BMI and the usual definitions of overweight and obesity are unlikely to fully quantify obesity-related disease burden in Thailand. Measures of obesity that incorporated assessment of fat distribution and methods of analysis that recognised the effects of adiposity on cardiovascular risk among individuals that do not reach standard definitions of overweight and obesity would be much more likely to achieve this.

Acknowledgements InterASIA Collaborative Group:

Thailand: Mahidol University, Bangkok-P. Suriyawongpaisal, S. Cheepudomwit, P. Loelekla, P. Srithara; Bangkok-P. Sariyaporn, P. Pongchoke, S. Jaiyavat, C. Nantawan, V. Kasikoson; North-S. Thamthitiwat, P. Siviroj, J. Suwanteerangkul; Northeast-P.
Tasanavivat, P. Kessomboon, S. Horas; South-V. Chongsuvivatwong, T. Yipintsoi, N. Apakupakul, W. Jirathamopas, W. Jintapakorn; Central-V. Kosulwat, A. Boonpraderm, A. Wongchanapai, C. Wanijjakul.

China: Chinese Academy of Medical Sciences, Beijing-D. Gu, X. Duan, G. Huang, X. Wu; Beijing-Y. Ma; F-L. Yu; Guangxi-Y. Chen; Hubei-L. Wang; Jiangsu-C. Yao; Jiling-L. Xu; Qinghai -P. Wang; Shandong-F. Chen; Shanxi-J. Mo; Sichuan - X. Wu.

United States: Tulane University, New Orleans, LA-J. He, P. Whelton, L. Bazzano, J. Chen, P. Muntner, K. Reynolds, X. Xin.

Australia: the George Institute for International Health, University of Sydney-N. Chapman, S. Colman, S. MacMahon, B. Neal, R. Stolk, M. Woodward. Steering Committee: J. He, P. Whelton, D. Glasser, D. Gu, S. MacMahon, B. Neal, R. Patni, R. Reynolds, P. Suriyawongpaisal, X. Wu, X. Xin.

Financial support-The InterASIA study was funded by a contractual agreement between Tulane University, LA, USA and Pfizer, NY, USA. Two researchers employed by Pfizer were members of the Steering Committee that designed the study. However, the study was conducted, analysed, and interpreted by the investigators independent of the sponsor.

Duality of interest - The authors have reported that no dualities of interest exist.

\section{References}

1. Sritara P, Cheepudomwit S, Chapman N et al (2003) Twelveyear changes in vascular risk factors and their associations with mortality in a cohort of 3499 Thais: the electricity generating authority of Thailand study. Int J Epidemiol 32:461-468

2. Tan CE, Ma S, Wai D, Chew SK, Tai ES (2004) Can we apply the National Cholesterol Education Program Adult Treatment Panel definition of the metabolic syndrome to Asians? Diabetes Care 27:1182-1186

3. Deurenberg P, Deurenberg-Yap M, Guricci S (2002) Asians are different from Caucasians and from each other in their body mass index/body fat percent relationship. Obes Rev 3:141-146

4. WHO Expert Consultation (2004) Appropriate body-mass index for Asian populations and its implications for policy and intervention strategies. Lancet 363:157-163

5. The International Collaborative Study of Cardiovascular Disease in Asia (InterASIA) (2003) Cardiovascular risk factor levels in urban and rural Thailand. Eur J Cardiovasc Prev Rehabil 10:249 257

6. Janssen I, Katzmarzyk PT, Ross R (2004) Waist circumference and not body mass index explains obesity-related health risk. Am J Clin Nutr 79:379-384

7. Wat NM, Lam TH, Janus ED, Lam KS (2001) Central obesity predicts the worsening of glycemia in southern Chinese. Int $\mathrm{J}$ Obes Relat Metab Disord 25:1789-1793

8. Ramachandran A, Snehalatha C, Vijay V (2004) Low risk threshold for acquired diabetogenic factors in Asian Indians. Diabetes Res Clin Pract 65:189-195

9. Tee ES (2002) Obesity in Asia: prevalence and issues in assessment methodologies. Asia Pac J Clin Nutr 11(Suppl 8):S694S701 